\title{
Efficient high-resolution TMS mapping of the human motor cortex by nonlinear regression
}

Numssen, Ole; Zier, Anna Leah; Thielscher, Axel; Hartwigsen, Gesa; Knösche, Thomas R.; Weise, Konstantin

\section{Published in:}

Neurolmage

Link to article, DOI:

10.1016/j.neuroimage.2021.118654

Publication date:

2021

Document Version

Publisher's PDF, also known as Version of record

Link back to DTU Orbit

Citation (APA):

Numssen, O., Zier, A. L., Thielscher, A., Hartwigsen, G., Knösche, T. R., \& Weise, K. (2021). Efficient highresolution TMS mapping of the human motor cortex by nonlinear regression. Neurolmage, 245, [118654]. https://doi.org/10.1016/j.neuroimage.2021.118654

\section{General rights}

Copyright and moral rights for the publications made accessible in the public portal are retained by the authors and/or other copyright owners and it is a condition of accessing publications that users recognise and abide by the legal requirements associated with these rights.

- Users may download and print one copy of any publication from the public portal for the purpose of private study or research.

- You may not further distribute the material or use it for any profit-making activity or commercial gain

- You may freely distribute the URL identifying the publication in the public portal 


\title{
Efficient high-resolution TMS mapping of the human motor cortex by nonlinear regression
}

\author{
Ole Numssen a,*, Anna-Leah Zier ${ }^{\mathrm{a}, \mathrm{d}}$, Axel Thielscher ${ }^{\mathrm{b}, \mathrm{c}}$, Gesa Hartwigsen ${ }^{\mathrm{a}}$, Thomas R. Knösche ${ }^{\mathrm{d}, \mathrm{e}}$, \\ Konstantin Weise ${ }^{\mathrm{d}, \mathrm{f}}$ \\ ${ }^{a}$ Lise Meitner Research Group Cognition and Plasticity, Max Planck Institute for Human Cognitive and Brain Sciences, Stephanstr. $1 a$, 04103 Leipzig, Germany \\ ${ }^{\mathrm{b}}$ Danish Research Centre for Magnetic Resonance, Centre for Functional and Diagnostic Imaging and Research, Copenhagen University Hospital Amager and Hvidovre, \\ Denmark \\ ${ }^{c}$ Technical University of Denmark, Center for Magnetic Resonance, Department of Health Technology, Kongens Lyngby, Denmark \\ dMethods and Development Group "Brain Networks", Max Planck Institute for Human Cognitive and Brain Sciences, Stephanstr. 1 a, 04103 Leipzig, Germany \\ e Technische Universität Ilmenau, Institute of Biomedical Engineering and Informatics, Gustav-Kirchhoff-Straße 2, 98693 Ilmenau, Germany \\ ${ }^{\mathrm{f}}$ Technische Universität Ilmenau, Advanced Electromagnetics Group, Helmholtzplatz 2, 98693 Ilmenau, Germany
}

\section{A R T I C L E I N F O}

\section{Keywords:}

Brain mapping

Finite element analysis

Motor cortex

Transcranial magnetic stimulation

Motor threshold

\begin{abstract}
A B S T R A C T
Transcranial magnetic stimulation (TMS) is a powerful tool to investigate causal structure-function relationships in the human brain. However, a precise delineation of the effectively stimulated neuronal populations is notoriously impeded by the widespread and complex distribution of the induced electric field.

Here, we propose a method that allows rapid and feasible cortical localization at the individual subject level. The functional relationship between electric field and behavioral effect is quantified by combining experimental data with numerically modeled fields to identify the cortical origin of the modulated effect. Motor evoked potentials (MEPs) from three finger muscles were recorded for a set of random stimulations around the primary motor area. All induced electric fields were nonlinearly regressed against the elicited MEPs to identify their cortical origin.

We could distinguish cortical muscle representation with high spatial resolution and localized them primarily on the crowns and rims of the precentral gyrus. A post-hoc analysis revealed exponential convergence of the method with the number of stimulations, yielding a minimum of about 180 random stimulations to obtain stable results.

Establishing a functional link between the modulated effect and the underlying mode of action, the induced electric field, is a fundamental step to fully exploit the potential of TMS. In contrast to previous approaches, the presented protocol is particularly easy to implement, fast to apply, and very robust due to the random coil positioning and therefore is suitable for practical and clinical applications.
\end{abstract}

\section{Introduction}

Transcranial magnetic stimulation (TMS) is a powerful non-invasive technique to modulate motor and cognitive functions in the human brain through induced electric fields. This provides insight into structure-function relationships at both group and individual level (e.g. Weise et al., 2020; Gomez et al., 2021). However, the precise cortical location at which an induced electric field initiates the behavioral effect remains unclear. Estimating the induced fields is key to address this (Weise et al., 2020; Gomez et al., 2021) but complex field distributions and interindividual variations hamper a precise cortical localization. For example, the regions which are initially activated for TMS-elicited motor evoked potentials (MEP) are still debated (Fig. 1). Several studies point to crown and lip stimulation in the caudal part of the dorsal premotor cortex (PMd, situated in BA6d) (Weise et al., 2020; Classen et al., 1998; Siebner, 2020; Dubbioso et al., 2021). Others point towards a direct stimulation of the hand representations (Fox et al., 2004; Krieg et al., 2013) in the primary motor cortex (M1, situated in BA4a/BA4p), inside the sulcal wall of the precentral gyrus. Localization efforts are further impeded by substantial inter-individual variation of the cortical geometry (Teitti et al., 2008; Diekhoff et al., 2011; Sarfeld et al., 2012; Ahdab et al., 2014; 2016; Vaalto et al., 2011).

Many previous mapping studies in the motor cortex (e.g. Neggers et al., 2004; Kleim et al., 2007; Ngomo et al., 2012; van de Ruit et al., 2015; Veldema et al., 2017) rely on center of gravity approaches and structured grids. This allows for the identification of those TMS coil

\footnotetext{
* Corresponding author: Max Planck Institute for Human Cognitive and Brain Sciences, Stephanstr. 1a, 04103 Leipzig, Germany.

E-mail address: numssen@cbs.mpg.de (O. Numssen).
} 


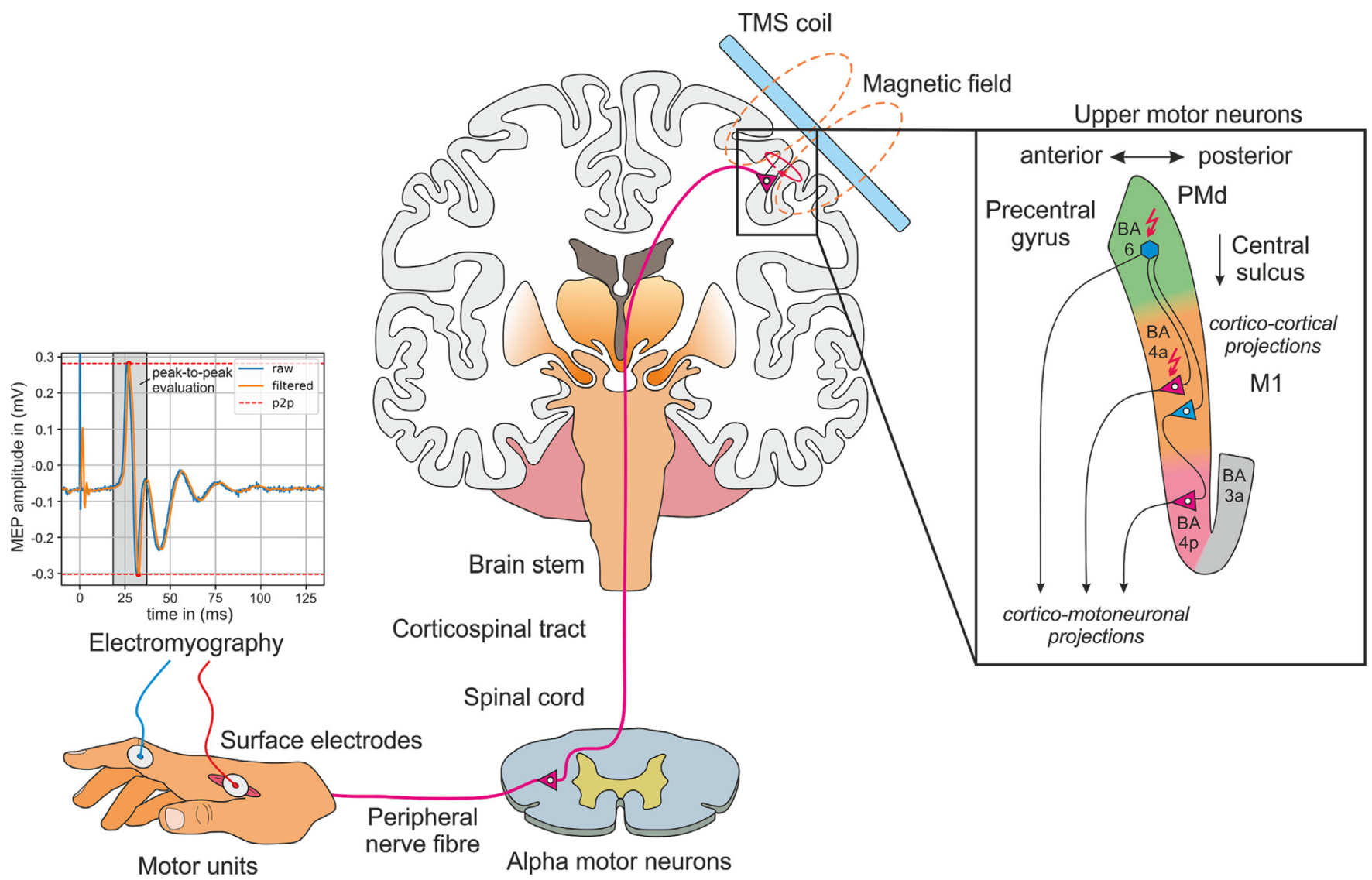

Fig. 1. Schematic representation of TMS experiments to measure motor evoked potentials (MEPs). A TMS coil is located at the scalp over the primary motor cortex (M1). A short electric field is generated via electromagnetic induction, yielding sufficiently stimulated neurons to depolarize. Subsequent muscle activation is quantified via electromyography. The cortical stimulation mechanics are currently still debated, with candidates including (i) direct stimulation of pyramidal output neurons in M1 (area BA4a/p); (ii) indirect depolarization of M1 output neurons via cortico-cortical projections from the dorsal premotor cortex (PMd, part of BA6d) to M1; and (iii) stimulation of pyramidal output neurons in PMd and direct cortico-motoneuronal projections to alpha motor neurons. Upper motor neuron details adapted from (Geyer et al., 1996).

positions on the head surface that yield the strongest effect. Frameworks have been proposed to reduce user-dependence (Tervo et al., 2020), relying on custom built TMS hardware to identify the optimal coil position in an automated way. These coil configurations can then be projected onto the cortex to estimate functionally relevant brain structures in a simplified manner (Krieg et al., 2013; Wassermann et al., 1996). However, these approaches can only provide a rough estimate of the effective stimulation site because information about the induced electric field is disregarded. Furthermore, no depth information is included and potential spatial offsets between the electric field peaks from a position directly under the coil are neglected.

Recently, localization methods have been improved by considering the interaction between estimated electric fields and observed effects, for example by superimposing multiple fields in an additive or multiplicative fashion (Opitz et al., 2013; Aonuma et al., 2018). Yet, such approaches suffer from the fundamental problem that the field maximum cannot be positioned freely throughout the cortex. Brain areas close to the TMS coil always receive higher field strengths than deeper located areas, leading to a strong bias in favor of superficial regions. To overcome this, Bungert et al. (2017) employed a statistical approach based on the resting motor threshold (rMT) for different coil orientations. Others (Laakso et al., 2018) investigated the influence of the coil position. We recently introduced an approach exploiting information from both coil positions and orientations (Weise et al., 2020). This linked the induced electric field strength to a measure quantifying the TMS effect (e.g., MEP amplitude). This relationship, the input-output curve (IOcurve), is evaluated at every cortical element of a fine mesh for a set of different coil positions and orientations. In contrast to previous studies, the approach was validated in an additional TMS experiment by explicitly testing whether stimulation with an optimized coil position and orientation for the proposed cortical locations yielded a larger effect than stimulation with deviating coil positions or orientations. This method was robust against measurement and tissue conductivity uncertainties. With only six optimal coil positions and orientations, resulting in about 600 TMS pulses, the localization problem could be solved. However, it remained unclear how to identify these positions and orientations. Here, we substantially advanced this method to allow arbitrary ('random') coil positions and orientations for each TMS pulse and thus providing a simple experimental protocol. This decreases the required number of stimulations for reliable localization, by virtue of increased electric field variability. We applied this method to 14 subjects to identify the somatotopic organization of three hand muscles within one TMS experiment. The proposed cortical muscle representations were validated in a second experimental session to show successful distinction of different finger muscle representations at the individual subject level. Based on an extensive convergence analysis, we provide metrics to evaluate the overall goodness of the proposed cortical mapping procedure.

\section{Materials and methods}

\subsection{Subjects}

Fourteen healthy, right-handed participants (seven females, age 2138 years) with a mean laterality index of $95.28(S D=7.93)$ accord- 
ing to the Edinburgh Handedness Inventory were recruited. Subject inclusion was in accordance with the safety guidelines for TMS studies (Rossi et al., 2021). Written informed consent was obtained from all participants prior to the examination. The study was performed according to the guidelines of the Declaration of Helsinki and approved by the local Ethics committee of the Medical Faculty of the University of Leipzig.

\subsection{Hardware setup}

TMS pulses were applied with a MagPro X100 stimulator (MagVenture, firmware Version 7.1.1) and a MCF-B65 figure-of-eight coil. Coil positioning was guided by a neuronavigation system (TMS Navigator, Localite, Germany, Sankt Augustin; camera: Polaris Spectra, NDI, Canada, Waterloo) and the coil positions were saved for each stimulation.

Electromyographic data (EMG) for three hand muscles were recorded from the subjects' right hand for each stimulation, namely the first dorsal interosseous (FDI), the musculus abductor digiti minimi (ADM), and the musculus abductor pollicis brevis (APB) using a standard bellytendon montage (Kleim et al., 2007). Electrodes were connected to a patient amplifier system (D-360, DigitimerLtd., UK, Welwyn Garden City; bandpass filtered from $10 \mathrm{~Hz}$ to $2 \mathrm{kHz}$ ), which was connected to an acquisition interface (Power1401 MK-II, CED Ltd., UK, Cambridge, $4 \mathrm{kHz}$ sampling rate). EMG recording was performed with Signal (CED Ltd., version 4.11). Subsequently, EMG data was lowpass filtered with a 6th order Butterworth filter (cutoff frequency: $500 \mathrm{~Hz}$ ). MEPs were calculated as peak-to-peak amplitudes in a time window 18 to $35 \mathrm{~ms}$ after the TMS pulse.

\subsection{Localization experiment}

Localization of the initial MEP target location was initially guided by group coordinates from (Mayka et al., 2006). We manually determined the rMT for the FDI and the corresponding coil position (Yousry, 1997). The rMT was defined as the minimum stimulator intensity yielding MEPs with an amplitude of at least $50 \mu \mathrm{V}$ in at least 5 out of 10 consecutive TMS pulses (Rothwell et al., 1999).

900-1100 single biphasic pulses were applied with a $5 \mathrm{~s}$ inter stimulus interval. Coil positions and angles were randomly selected for each stimulation to sample electric field distributions and corresponding MEPs for different induced electric fields. The coil center positions were restricted to a circular area of $2 \mathrm{~cm}$ radius around the estimated M1 location (Fig. 2) and the angles to approximately $\pm 60^{\circ}$ in relation to the estimated optimal coil angle. The stimulation area definition proved sufficient to yield MEPs amplitudes from the upper limit of the I/O curve at the estimated M1 stimulation site and small or no MEPs at the periphery for a fixed stimulator intensity. This intensity, about $150 \%$ of the estimated rMT, was individually predetermined to stay in a comfortable range. Experimenters were instructed to evenly sample the determined area such that all parts of the stimulated area were visited several times throughout the experimental session to guard against possible sequential effects. A second experimenter monitored the EMG signal from all three muscles to identify any muscle pre-activation in which case the stimulation was paused.

\subsection{Numerical simulations of the induced electric field}

Electric field calculations were conducted for each pulse (SimNIBS v3.1, (Saturnino et al., 2019; Thielscher et al., 2015)) with highresolution anisotropic finite element models (FEMs). Magnetic resonance imaging (MRI) scans were acquired on a 3T MRI scanner (Siemens Verio or Skyra) with a 32 channel head coil using the same acquisition parameters as before (Weise et al., 2020). T1 and T2 images were used for tissue type segmentation. Conductivity tensors in gray and white matter were reconstructed from diffusion weighted images using the volume normalized mapping approach (dwi2cond, https:// simnibs.github.io/simnibs/build/html/documentation/command_line/ dwi2cond.html, (Güllmar et al., 2010)). Individual head models were generated using the headreco pipeline (Nielsen et al., 2018) utilizing SPM12 (https://www.fil.ion.ucl.ac.uk/spm/software/spm12/, (Penny et al., 2011)) and CAT12 (http://www.neuro.uni-jena.de/cat/, (Gaser et al., 2021)). The final head models were composed of $\sim 3.4 \cdot 10^{6}$ nodes and $\sim 18.5 \cdot 10^{6}$ tetrahedra (average volume: $\sim 0.15 \mathrm{~mm}^{3}$ in the cortex). Six tissue types were included with the following conductivity estimates: white matter $\left(\sigma_{W M}=0.126 \mathrm{~S} / \mathrm{m}\right)$, gray matter $\left(\sigma_{G M}=0.275 \mathrm{~S} / \mathrm{m}\right)$, cerebrospinal fluid $\left(\sigma_{C S F}=1.654 \mathrm{~S} / \mathrm{m}\right)$, bone $\left(\sigma_{B}=0.01 \mathrm{~S} / \mathrm{m}\right)$, skin $\left(\sigma_{S}=0.465 \mathrm{~S} / \mathrm{m}\right)$, and eyeballs $\left(\sigma_{E B}=0.5 \mathrm{~S} / \mathrm{m}\right)$ (Thielscher et al., 2015; Wagner et al., 2004). See (Saturnino et al., 2019) for FEM details.

A region of interest (ROI) was defined around the handknob area (FreeSurfer, http://surfer.nmr.mgh.harvard.edu/, (Fischl et al., 1998; Dale et al., 1999)) based on the fsaverage template. This covered parts of somatosensory cortex (BA1, BA3), primary motor cortex M1 (BA4), and dorsal premotor cortex PMd (BA6). The HCP-MMP parcellation was used (Glasser et al., 2016) to visualize the border between PMd and M1. All analyses were performed on the gray matter midlayer, halfway between gray and white matter surfaces.

\subsection{Determining the site of effective stimulation}

The core concept of the proposed method is illustrated in Fig. 2. We assume i) a sigmoidal relationship between either the electric field magnitude or a directional field component and MEP amplitude at the cortical location where the electric field elicits the observed MEP, based on our (Weise et al., 2020) and others' (Goetz et al., 2019) previous work; ii) that different combinations of coil position, coil orientation, and stimulation intensity can generate the same electric field in a particular neuronal population; iii) that for each muscle exactly one focal cortical area in the primary motor cortex is functionally relevant for MEP evocation by TMS (Aberra et al., 2020; Siebner, 2020). Exploiting these assumptions, the site of effective stimulation can be pinpointed by fitting cortical I/O curves (Weise et al., 2020), relating the local electric field to the MEP amplitude, and subsequently quantifying their goodness-of-fit. The goodness-of-fit would be highest at the cortical site that houses the relevant neuronal populations. Fitting can be performed for different components of the electric field vector, such as its magnitude $(|E|)$, its projection onto the cortical surface normal $\left(\left|E_{\perp}\right|\right)$, its projection into the cortical tangential plane $\left(\left|E_{||}\right|\right)$, and any other quantities derived thereof. Based on findings from experimental (Weise et al., 2020) and modeling approaches (Aberra et al., 2020), we focus on the field magnitude. Fitting can be performed with different functions. We compared (i) standard linear regression, (ii) nonlinear regression using a sigmoidal function, and (iii) nonlinear regression using a log-transformed sigmoidal function.

(i) In standard linear regression, a linear relationship between electric field intensity $x_{i j}$ of stimulation $\mathrm{i}$ at the cortical element $\mathrm{j}$ and the MEP amplitude $y_{i}$ is assumed:

$\tilde{y}_{i, j}=\beta_{0, j}+\beta_{1, j} x_{i, j}, 1 \leq i \leq N_{T M S}, 1 \leq j \leq N_{\text {elms }}$

where $N_{T M S}$ and $N_{\text {elms }}$ denote the total number of applied TMS pulses and number of elements in the examined region of interest (ROI) respectively. $\beta_{0}$ and $\beta_{1}$ are estimated in every ROI element $j$. This approach is the cheapest in terms of computational cost but neglects the characteristic sigmoidal shape of the input-output curve observed in the motor cortex.

(ii) A sigmoidal input-output function provides more physiological plausibility:

$\tilde{y}_{i, j}=y_{0}+\frac{a_{j}-y_{0}}{1+e^{-r_{j}\left(x_{i, j}-x_{0, j}\right)}}, 1 \leq i \leq N_{T M S}, 1 \leq j \leq N_{e l m s}$

where $a$ is the saturation amplitude, $r$ is the slope, $x_{0}$ is the location of the turning point on the abscissa, and $y_{0}$ denotes the offset. The offset 


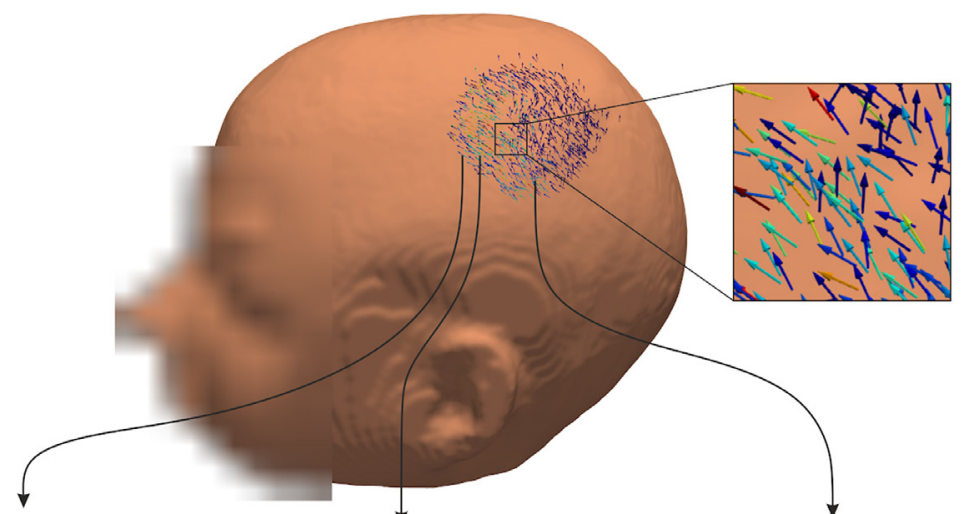

Stimulation 1

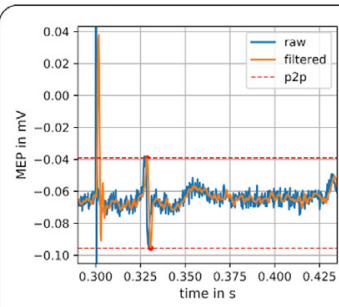

Electric field

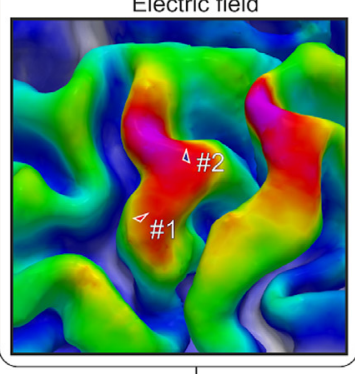

$\downarrow$

ROI element \#1

(high goodness of fit)

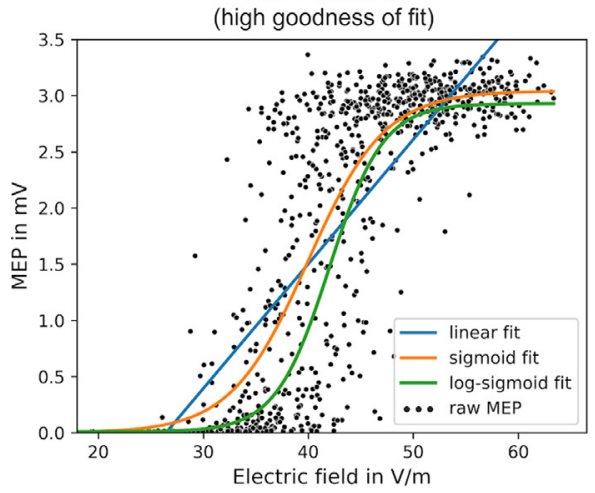

Stimulation 2

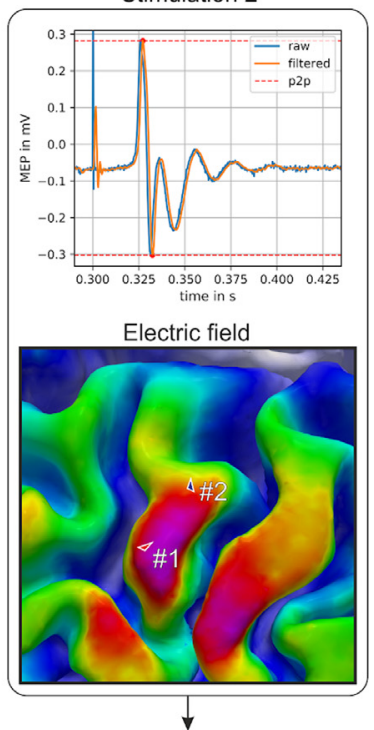

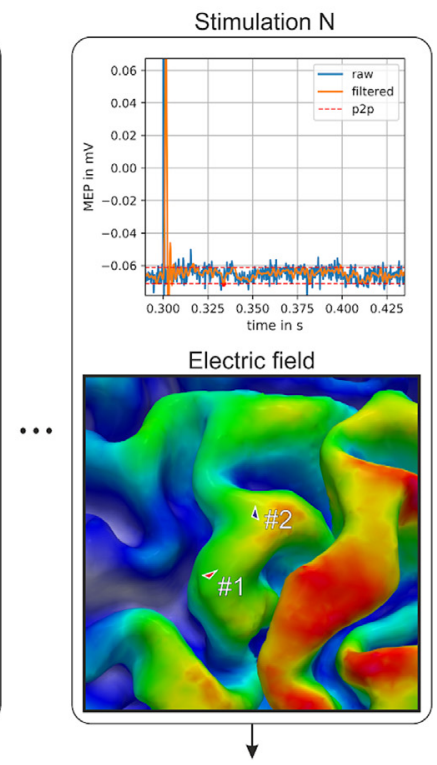

ROI element \#2 (low goodness of fit)

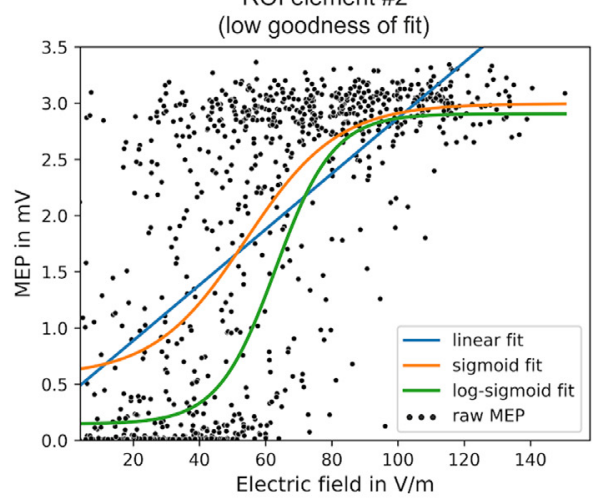

Fig. 2. General principle of the localization approach. Top: $N$ TMS pulses are applied at random coil locations and orientations around the M1 area. Stimulator intensity is calibrated such that large MEPs are elicited at the center of the examined area. For each pulse, a motor evoked potential (MEP) is recorded with muscle EMG and the induced electric field is calculated. Middle: For each cortical element, the electrical field quantity of interest (e.g. its magnitude) is regressed on the MEP amplitude. Bottom: A goodness-of-fit map identifies the most probable origin of the MEPs.

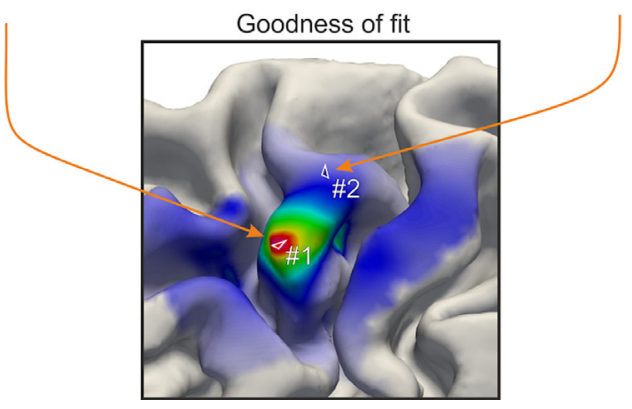


parameter $y_{0}$ results from measurement noise. We estimated $y_{0}$ from baseline EMG data in the absence of any stimulus (Alavi et al., 2019) to reduce the number of free parameters.

(iii) EMG data from this domain are typically heteroscedastic and log-transformation may be applied to equalize variance across the range of MEP magnitudes (Goetz et al., 2014, 2019; Peterchev et al., 2013; Nielsen, 1996). Here, we used a sigmoidal function of the following type:

$\tilde{y}_{i, j}=\log _{10}\left(y_{0}+\frac{a_{j}-y_{0}}{1+e^{-r_{j}\left(x_{i, j}-x_{0, j}\right)}}\right), 1 \leq i \leq N_{T M S}, 1 \leq j \leq N_{e l m s}$

The non-linear functions were fitted in every ROI element using the Levenberg-Marquardt algorithm.

We assessed the element-wise goodness-of-fit by means of the coefficient of determination $R^{2}$ :

$R_{j}^{2}=1-\frac{\operatorname{VAR}\left(y-\tilde{y}_{j}\right)}{\operatorname{VAR}(y)}, 1 \leq j \leq N_{\text {elms }}$,

where $y$ and $\tilde{y}_{j}$ are vectors containing measured and fitted MEPs, respectively. The better the fit, the closer $R^{2}$ is to unity.

The sign of the normal component depends on the field's direction. To prevent the confounding of possibly differing neuronal activation mechanisms we separated the electric fields by their signs. Specifically, we performed one initial linear fit in each element for both datasets, i.e. positive and negative field components, and determined the slopes and associated $p$ values. Only datasets with $p>0.001$ and a positive slope for positive electric field components or a negative slope for negative electric field components were considered for further analyses. The dataset yielding the higher $R^{2}$ score was chosen for the final non-linear fit.

The proposed method is principally equivalent to the curve shift approach proposed previously (Weise et al., 2020). However, in our approach, curve fitting is done element-wise in the E-MEP space leveraging information from every single TMS pulse, in contrast to the conditionwise approach implemented before. The procedure yields one cortical map of $R^{2}$ scores per muscle, quantifying the probability of generating MEPs for each cortical element.

\subsection{Validation experiment}

After identifying the neuronal population (the 'hotspot') that appears to underlie the observed MEP, i.e. that yielded the highest $R^{2}$ score, we validated this finding in a second experimental session. Based on field simulations we selected the coil position/orientation that induced the strongest electrical field at the hotspot. This was done for each of the three muscle representations and for each subject individually. To verify if stimulation of the proposed hotspot does indeed lead to the largest effect, rMTs were acquired for these optimal coil positions/orientations and for adjacent ones. Optimal coil positions/orientations were determined with an extensive search procedure, comparing electrical fields magnitudes of 4852 coil configurations at the hotspot area (search radius $=20 \mathrm{~mm}$, spatial resolution $=2.5 \mathrm{~mm}$, search angle $=180^{\circ}$, angle resolution $=7.5^{\circ}$ ). The optimization routine is implemented in SimNIBS v3.1 (https://simnibs.github.io/simnibs) and has been described before ((Weise et al., 2020); Supplementary Material). To determine the true rMT, single biphasic pulses with $5 \mathrm{~s}$ interstimulus interval were applied. The same figure-of-eight coil as before was used here. The rMTs for optimal coil configurations were compared to rMTs obtained for six adjacent coil configurations. Two coil configurations shared the same coil center with the optimal one but differed in their coil orientation $\left(-45^{\circ}\right.$ and $+45^{\circ}$ ) and four coil configurations shared the optimal coil orientation, but were shifted $7.5 \mathrm{~mm}$ into superior, posterior, inferior, or anterior directions.

\subsection{Convergence analysis}

To identify the minimum number of pulses needed to carry out the cortical localization, we studied random subsets of the realized stimu- lations, from $n=10$ to all available (900-1100) stimulations. We drew 100 independent subsets for each subject to approximate the robustness.

We assessed two metrics: i) the normalized root mean square deviation (NRMSD) as a measure of overall shape similarity of the resulting cortical map and ii) the geodesic distance to quantify an estimate of the accuracy of the hotspot identification. Convergence for both was quantified against the full set of stimulations as well as against the previous solution from $n-1$ stimulations. The former yields a proxy for the (unknown) ground truth, whereas the latter quantifies the magnitude of change from one stimulation to the next. This stability measure can be used in online analyses to construct a stop criterion, as well as for post-hoc evaluation of the overall goodness of the mapping procedure.

The NRMSD between the $R_{n}^{2}$ map for $n$ stimulations and the reference map $R_{r e f}^{2}$ was calculated as:

$\varepsilon_{n, r e f}^{R^{2}}=\frac{\sqrt{\frac{1}{N_{e l m s}} \sum_{i=1}^{N_{\text {elms }}}\left(R_{i, n}^{2}-R_{i, r e f}^{2}\right)^{2}}}{\max \left(R_{r e f}^{2}\right)-\min \left(R_{r e f}^{2}\right)}$

The geodesic distance $\varepsilon_{n, r e f}^{\delta}$ between the maxima locations of $R_{n}^{2}$ and $R_{r e f}^{2}$ was calculated with tvb-gdist 2.1.0 (github.com/the-virtual-brain/tvbgdist). To estimate the generalizability of our results we carried out nonparametric permutations tests across the stimulation subsets. These were performed against the full set solution. Based on this, the lower bound of the number of stimulations, which was needed to reach $<5 \%$ error NRMSD, could be derived. For the geodesic distance a criterion of $<$ $5 \mathrm{~mm}$ was chosen.

\section{Results}

\subsection{Localization}

For each subject, we calculated the cortical mapping with linear, sigmoidal, and log-transformed sigmoidal functions to identify the representations of APB, FDI, and ADM. Detailed results are presented in Fig. 3 for one subject considering the electric field magnitude (see Fig. S1 and S2 for the tangential and normal component, respectively). The general shape of the normalized $R^{2}$ maps are very similar across different fitting functions. The computation time for sigmoidal mappings was significantly shorter than for mappings with log-transformed sigmoids. Thus, we used sigmoidal functions throughout the rest of the analyses as this function type provides a well-balanced compromise in terms of mapping accuracy and computation efficacy. Table S1 provides peak $R^{2}$ values for all methods per subject.

Mapping results for all 14 subjects considering the electric field magnitude are shown in Fig. 4 (see Fig. S3 and S4 for the tangential and normal component, respectively). The $R^{2}$ hotspots are primarily located on the gyral crown and rim of the precentral gyrus. Representations of FDI and APB were found to be located closer to each other than to ADM, which was generally situated superior to them. For one subject, the muscle representations were located in M1, whereas for 13 subjects, they were located in caudal PMd. See Table 1 for group average results.

The localization based on the different field components identified similar cortical regions. The field's magnitude yielded significantly higher goodness-of-fit values than the tangential component ( $Z=249$, $p=0.0113$ ) and significantly higher goodness-of-fit values than the normal component $(Z=289, p=0.0422$ ). For both comparisons a Wilcoxon signed-rank test was performed due to non-normality of the data.

\subsection{Validation}

After identifying the cortical digit hotspots, we determined optimal coil positions to stimulate these hotspots and measured rMTs for these and for adjacent coil configurations to validate the mapping results. At the digit hotspots the adjacent coil positions yielded about $94 \%$ of the 

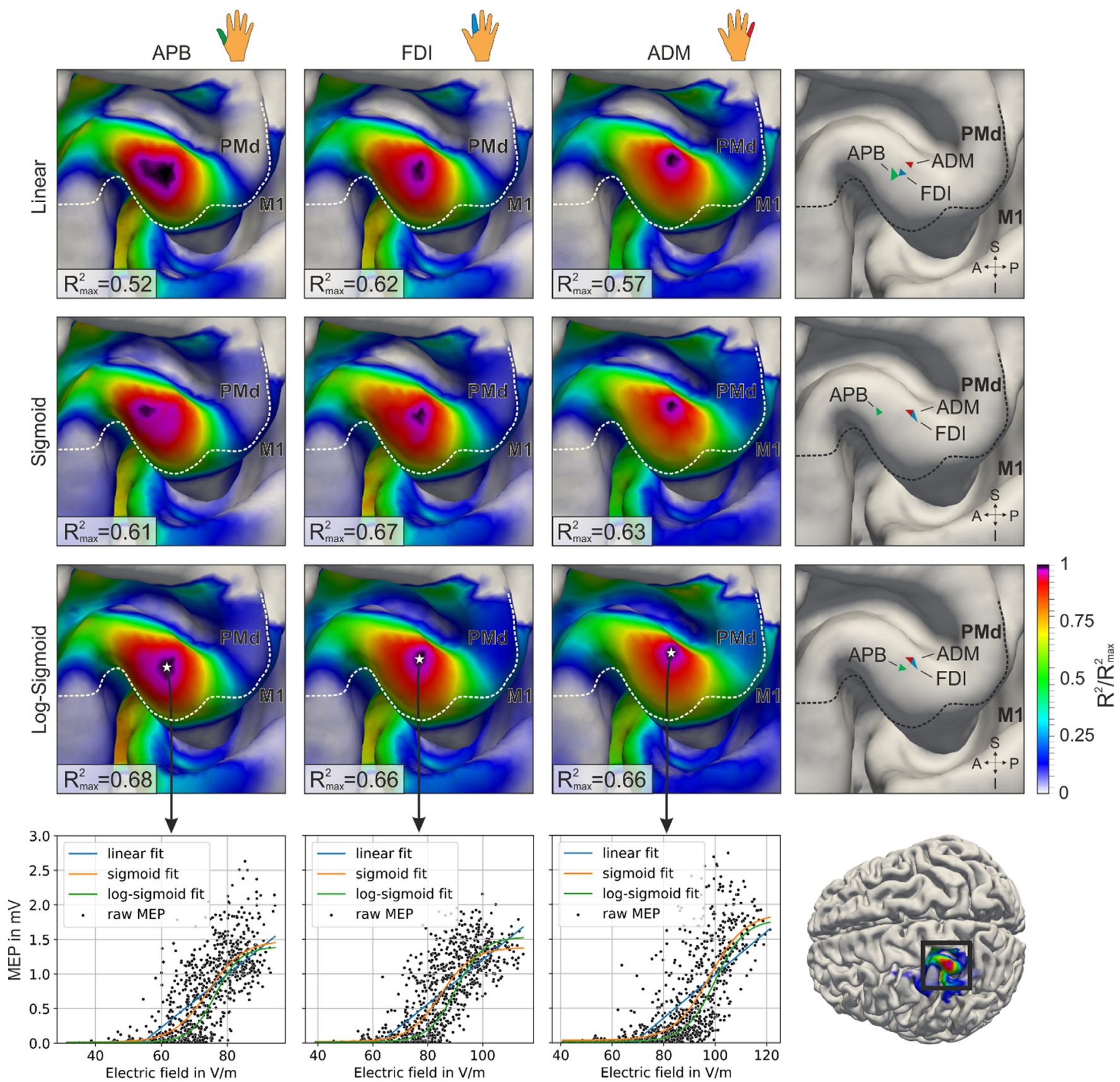

Fig. 3. Log-sigmoidal fitting exhibits an optimal yield-cost tradeoff. The first three columns show the normalized coefficient of determination $\left(R^{2}\right)$ for APB, FDI, and ADM, respectively, considering the magnitude of the electric field for subject S12. Maximum absolute values range between 0.3 for linear regression and 0.7 for non-linear regression. The last column highlights the identified hotspots for the three digits. Mapping results for linear, sigmoidal, and log-transformed sigmoidal functions are of a similar shape (rows). The boundary between dorsal premotor cortex PMd/BA6 and the primary motor cortex M1/BA4 are determined with the HCP-MMP atlas (Glasser et al., 2016). Function fits for optimal elements are characterized by a clear relation between the induced electric field strength and the evoked motor potentials (bottom). $R^{2}$.scores are normalized to the subject- and muscle-wise maximum value $R_{\max }^{2}$.

Table 1

Group-average results for the three finger muscles

\begin{tabular}{llllll}
\hline Muscle & MNI x & MNI y & MNI z & Relative MEP size & Absolute MEP size \\
\hline ABP & $-34.70( \pm 2.51)$ & $-13.99( \pm 3.12)$ & $66.57( \pm 2.88)$ & $86 \%$ & $4.88 \mathrm{mV}( \pm 0.59 \mathrm{mV})$ \\
FDI & $-34.19( \pm 2.59)$ & $-14.33( \pm 3.40)$ & $66.83( \pm 3.00)$ & $100 \%$ & $5.68 \mathrm{mV}( \pm 0.66 \mathrm{mV})$ \\
ADM & $-32.72( \pm 3.77)$ & $-16.09( \pm 3.30)$ & $68.03( \pm 3.11)$ & $72 \%$ & $4.10 \mathrm{mV}( \pm 0.59 \mathrm{mV})$ \\
\hline
\end{tabular}

Note: Coordinates in MNI space ( \pm standard error of mean). The last two columns show the relative size of the maximum MEP elicited and the mean ( \pm standard error of mean) across subjects for subjects 2 - 14 . 

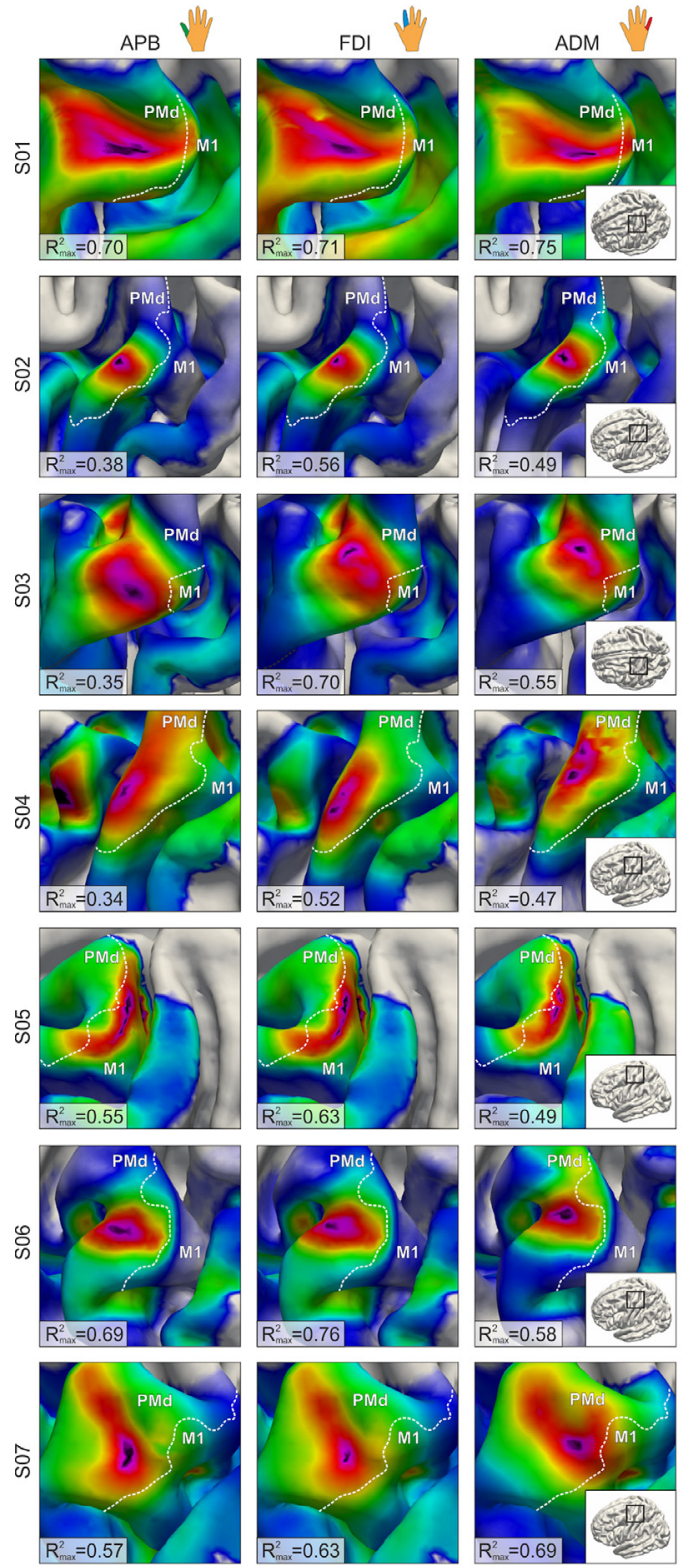
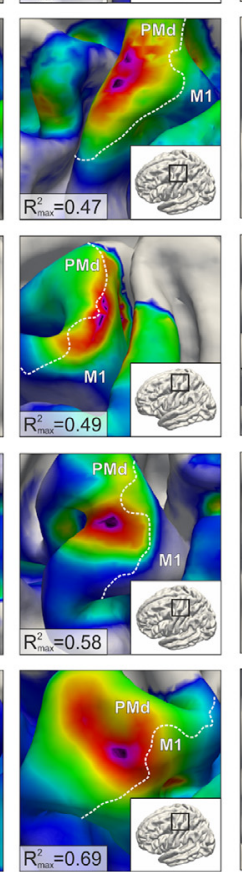
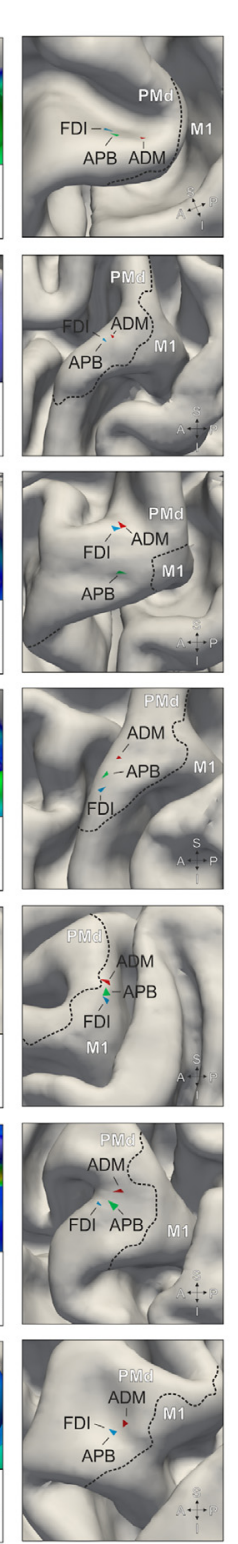
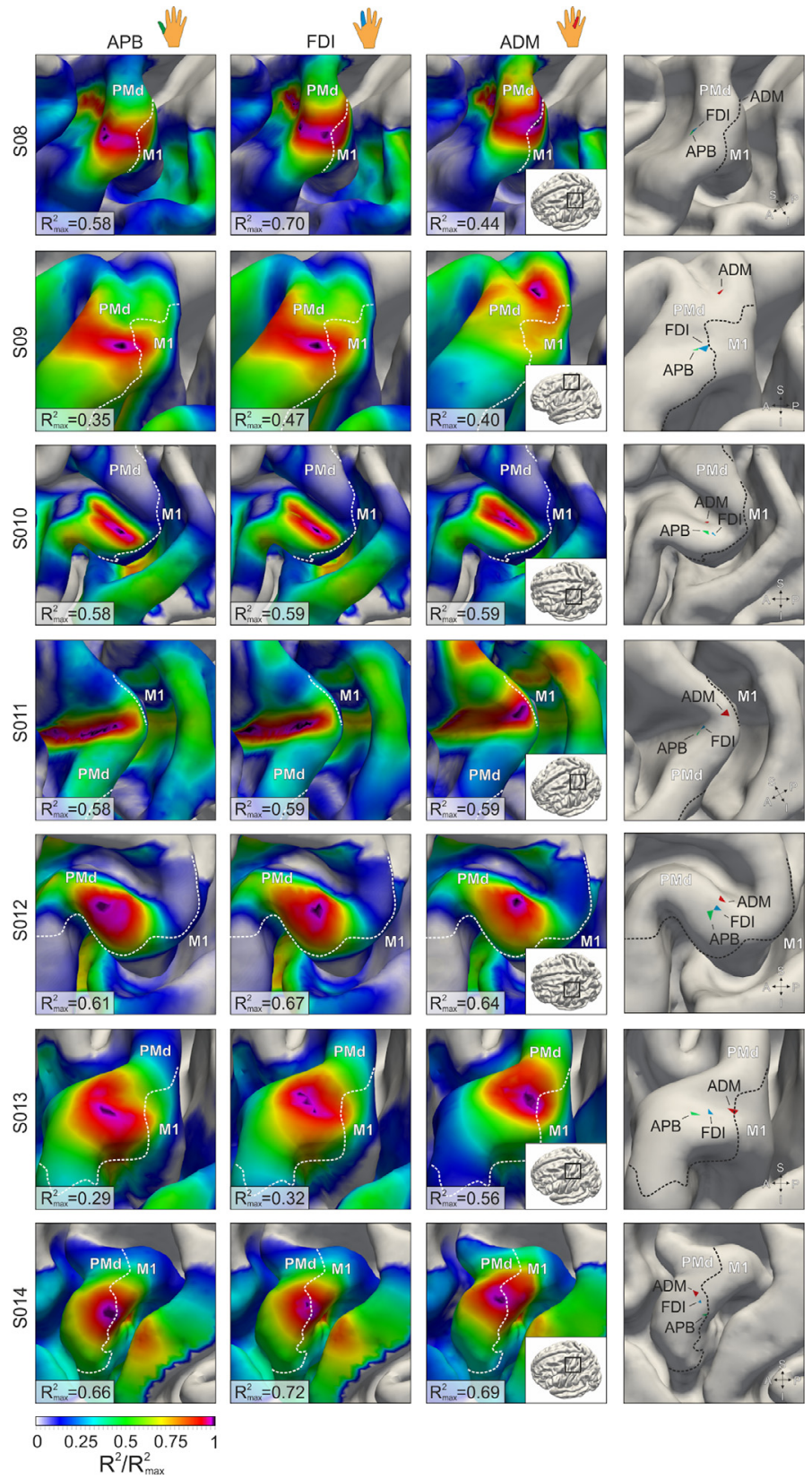
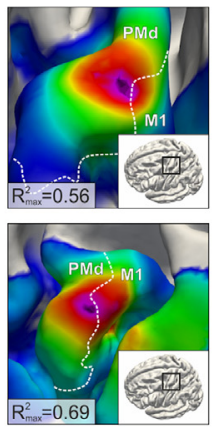

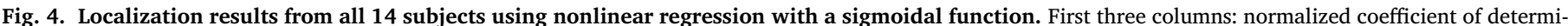

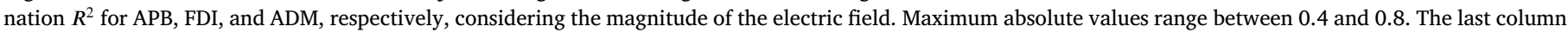

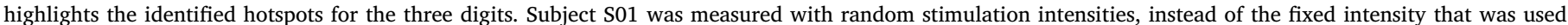

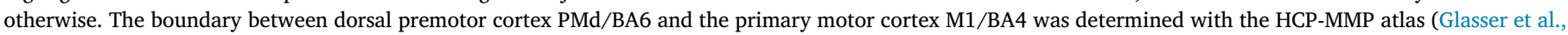
2016). $R^{2}$ scores are normalized to the subject- and muscle-wise maximum value $R_{\max }^{2}$.

stimulation strength induced by the optimal coil position and the rotated coil position yielded about $89 \%$, respectively (see Table $\mathrm{S} 1$ for details). This translates to absolute field differences of about $3 \mathrm{~V} / \mathrm{m}$ for the shifted stimulation conditions and to differences of about $6 \mathrm{~V} / \mathrm{m}$ for the rotated stimulation conditions respectively. Of the 14 initial subjects, nine participated in the validation study (Fig. 5). In the majority of cases the rMT for the optimal coil position was the lowest. Because data was not distributed normally (Shapiro-Wilk test: $W=0.91299, p=4.003 e-09$ ) one Mann-Whitney test was performed for each muscle. Resting MTs at adjacent positions were significantly higher than rMTs from the optimal, pre-computed coil positions and orientations (APB: $W=414$, $p=3.459 e-04 ;$ FDI: $W=455, p=1.47 e-05$; $\mathrm{ADM}: W=423$, $p=1.899 e-04)$.

\subsection{Convergence analysis}

For 100 random sequences, we calculated the mapping ranging from $n=10$ to all available stimulations $(N)$. NRMSD and geodesic distance converged exponentially, tested against all available stimulations $(N)$ and against the previous solution $(n-1)$. The variance across these localizations was large only until about $n=50$ and then quickly decreased. The convergence properties are comparable between subjects for the NRMSD metric (Fig. 6), and distinctively more pronounced for the geodesic distance (Fig. 7). Across subjects and metrics, localizing FDI was feasible with fewer stimulations than localizing ADM and APB.

With non-parametric bootstrapping tests we identified the number of stimulations required to localize within a $95 \%$ confidence interval. Tests 


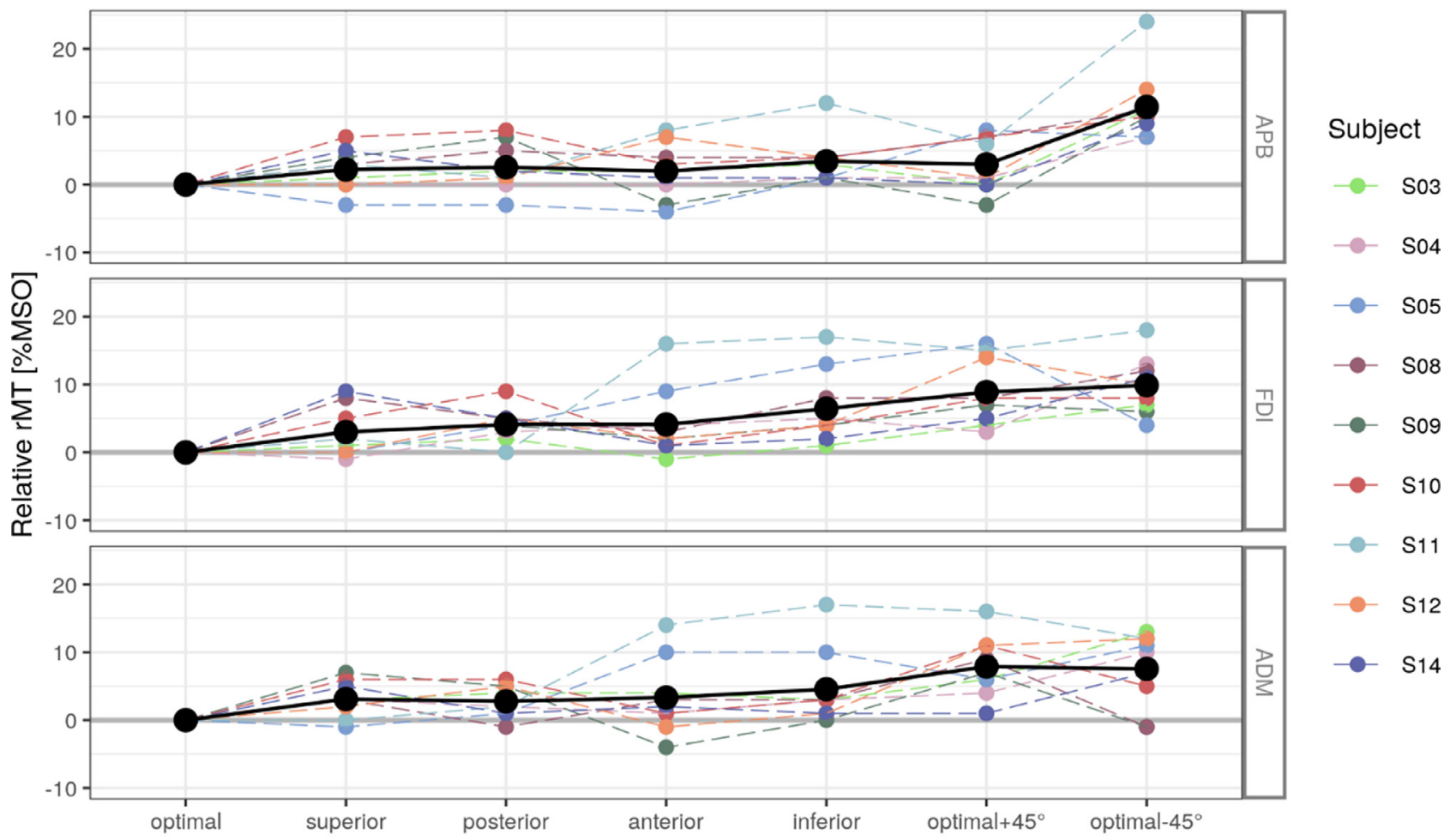

Coil Position

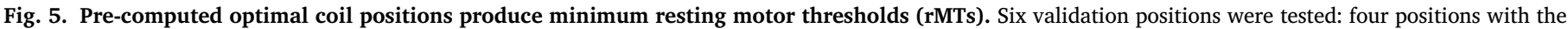

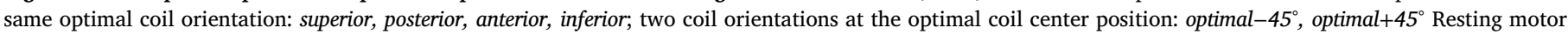

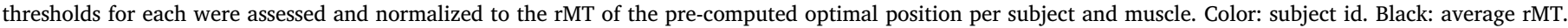

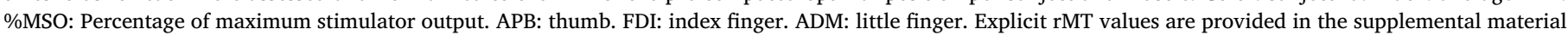
Table S3.

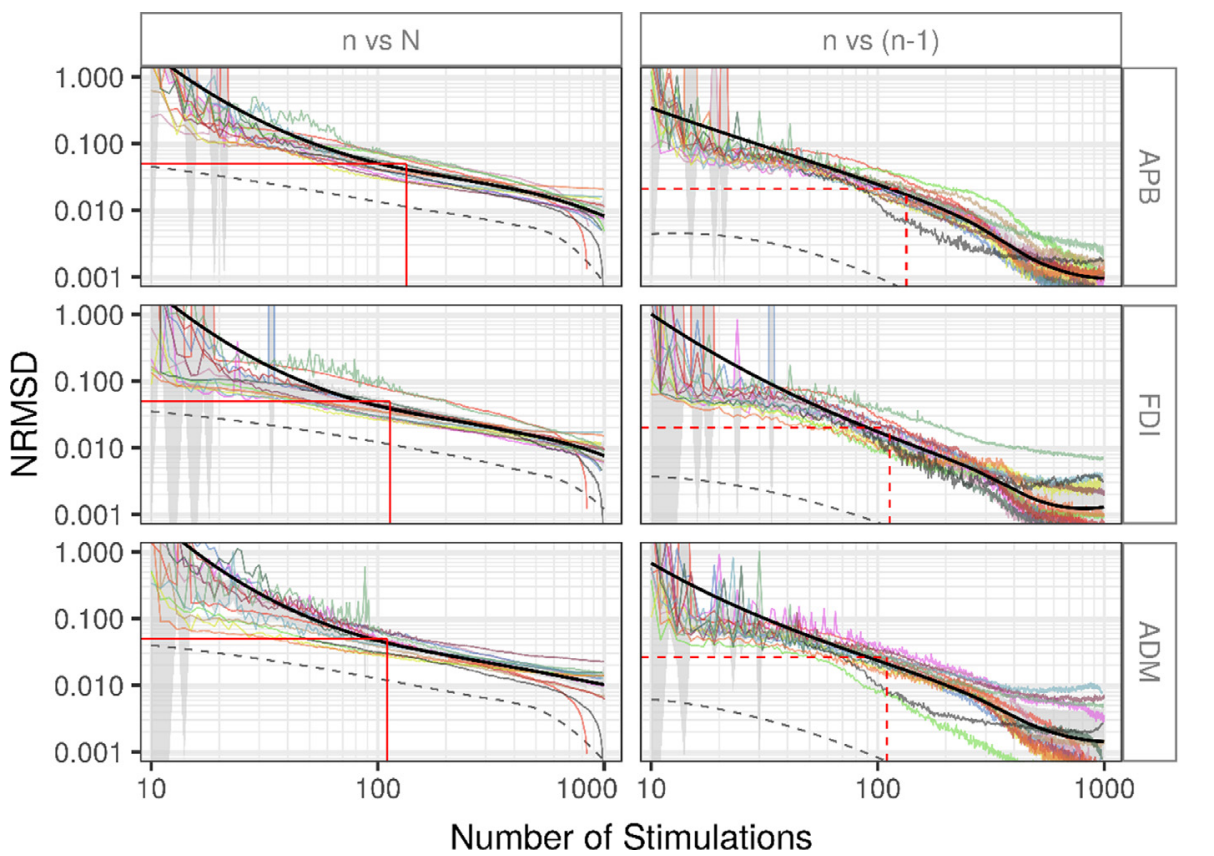

Subject

- S01

- S02

- $\mathrm{S} 03$

- $\$ 04$

- S05

- S06

- $\mathrm{S} 07$

- S08

- 509

- $\mathrm{S} 10$

- $S 11$

- $\mathrm{S} 12$

- $\$ 13$

- $\$ 14$

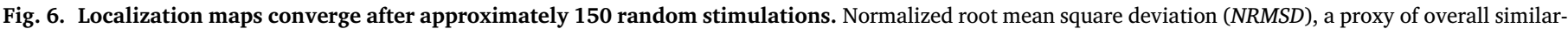

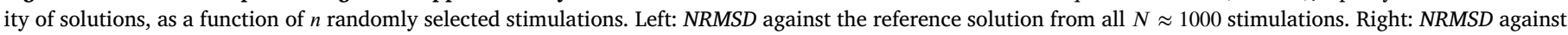

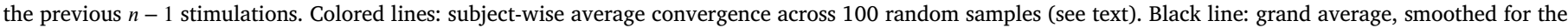

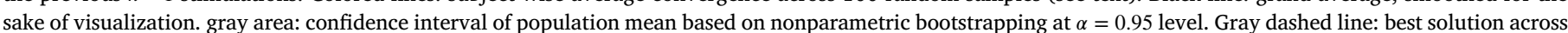

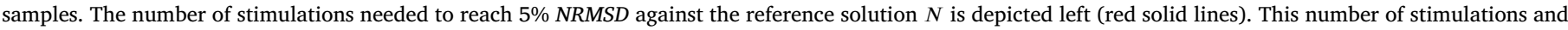

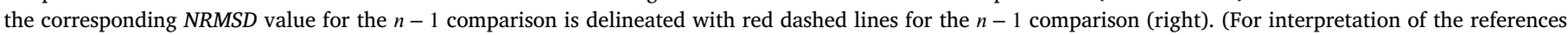
to color in this figure legend, the reader is referred to the web version of this article.) 


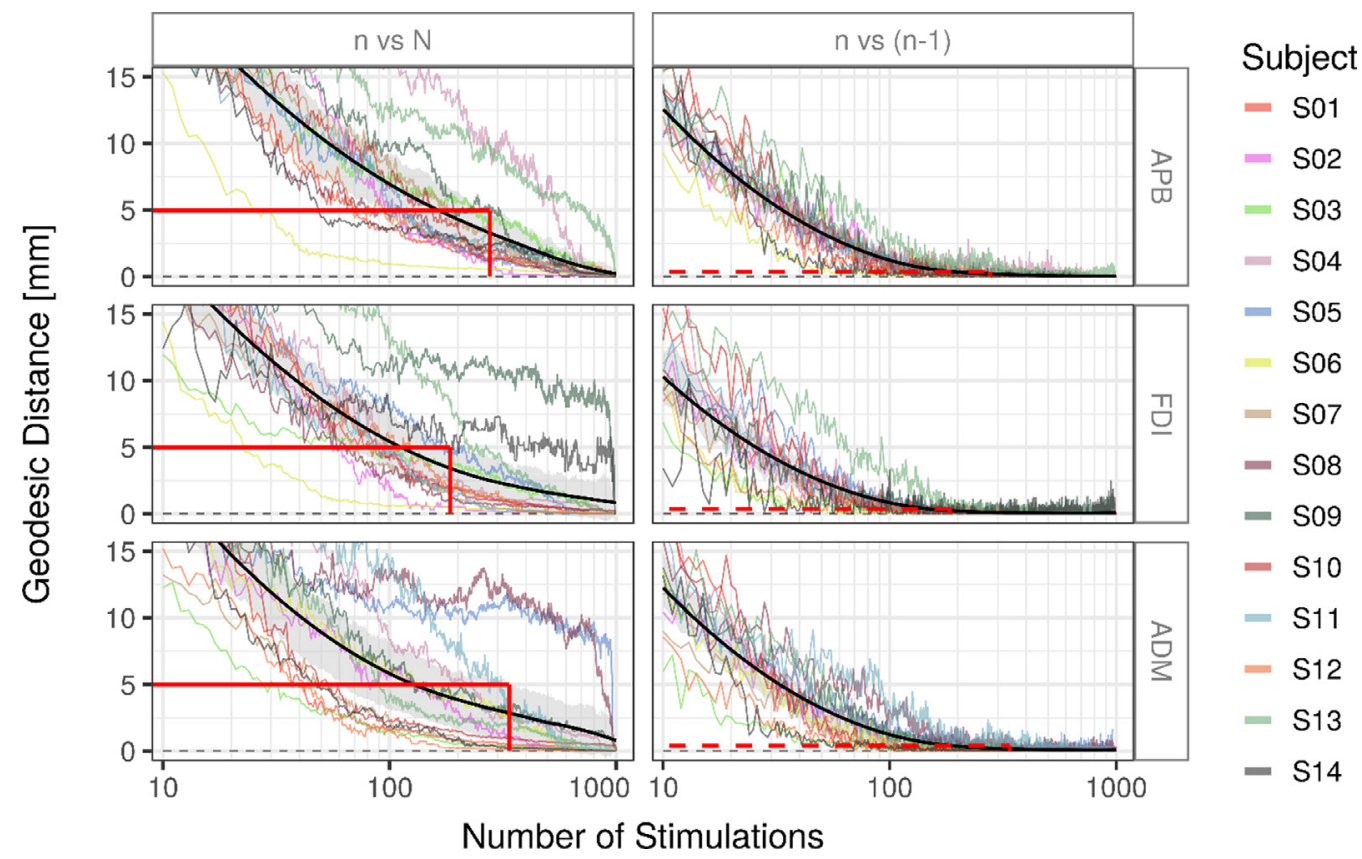

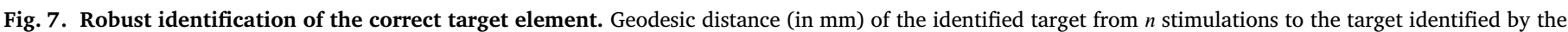

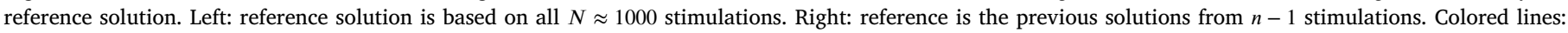

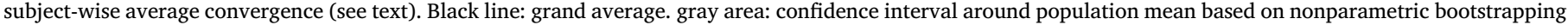

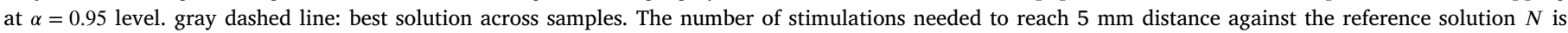

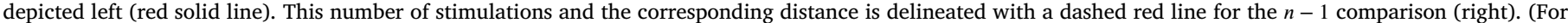
interpretation of the references to color in this figure legend, the reader is referred to the web version of this article.)

were performed for the comparison against all stimulations as a proxy for the ground truth. 134, 113, and 110 stimulations are necessary for $\mathrm{APB}, \mathrm{FDI}$, and ADM, respectively, for the overall similarity of $R^{2}$ maps (NRMSD) to reach an error $<5 \%$. This corresponds to NRMSD values of $2.0 \%, 2.0 \%$, and $2.6 \%$ (APB, FDI, ADM) for comparisons against the previous solution $(n-1)$. Correct cortical targets can be pinpointed within $5 \mathrm{~mm}$ with 277, 185, and 338 (APB, FDI, ADM) stimulations as quantified by the geodesic distance. This corresponds to $0.36 \mathrm{~mm}, 0.37 \mathrm{~mm}$, and $0.40 \mathrm{~mm}$ distance when comparing against the previous solution $(n-1)$.

See Vid. 1 for a video demonstration of the FDI $R^{2}$ map convergence behavior for a representative subject.

\section{Discussion}

We propose an efficient and high-resolution method to identify structure-function relationships with TMS. Here, we applied this method to map the individual somatotopy of several hand muscle representations in the primary motor cortex. This approach links information about the induced electric fields at the cortex to a modulated experimental outcome and relies on the variance across induced electric fields from multiple different stimulation sites. It is easy to implement in a standard TMS laboratory because the localization does not depend on predetermined sensitive coil positions and orientations and is therefore more suitable for practical and clinical use than previously proposed mapping approaches.

Specifically, we applied TMS pulses with randomly chosen coil positions and orientations around the larger primary motor region and recorded motor evoked potentials (MEPs) from several hand muscles. Subsequent electric field modeling and biophysical informed analyses allowed us to identify the associations between electric field magnitude and MEP size in the cortex. In line with previous work (Weise et al., 2020; Aberra et al., 2020), hotspots were found on the crowns and rims of the precentral gyrus between BA6 and BA4 for the magnitude and the tangential component of the electric field. For the normal compo- nent similar locations were identified, although with significantly lower goodness-of-fit.

Based on our results, we reason that TMS either evokes muscle activity through direct cortico-motoneuronal pyramidal neurons from the caudal part of PMd (PMdc) (Dum and Strick, 2002) or indirectly via cortico-cortical premotor-motor (PMdc-to-BA4a/p) projections (Ninomiya et al., 2019). This is supported by studies reporting that TMS during periods of high surface negativity measured by EEG leads to higher MEP amplitudes (Schaworonkow et al., 2018; Zrenner et al., 2018; Bergmann et al., 2019). This surface negativity may specifically stem from apical dendrites of neurons with a radial cortical orientation (Triesch et al., 2015), which are located in the crown of the precentral gyrus. Since the exact relationship between oscillations and corticospinal excitability remains unknown, future work on accurate neuronal network models is necessary to address this question.

The observed geodesic distances between finger representations are consistent with recent high-resolution functional MRI studies (Huber et al., 2020). Moreover, the general somatotopic organization of hand muscle representations in the primary motor cortex is in accordance with previous work, with APB being located infero-lateral to ADM (Wilson et al., 1993; Martuzzi et al., 2014) and ADM superior to FDI (Dubbioso et al., 2021; Raffin et al., 2015; Raffin and Siebner, 2019; Dubbioso et al., 2017). The observed substantial variability between subjects is also consistent with previous studies (Goldsworthy et al., 2016).

We extensively assessed the convergence behavior of the presented mapping method. Thereby, we were able to estimate a lower bound for the number of random stimulations needed to achieve robust somatotopic maps. About 150 pulses from random coil positions and orientations suffice to obtain robust cortical maps. This yields a mapping duration of less than $15 \mathrm{~min}$. Pinpointing single muscle representations, in contrast to an overall cortical probability map, requires between 190 (FDI) and 340 (ADM) pulses on average with significant inter-subject variability. Information about the convergence properties enables the quantification of the overall quality of a mapping. Specifically, the nor- 
malized root mean square deviation between all realized stimulations versus all but one stimulations $(n-1)$ should be as low as $2 \%$ to assert valid mapping results. It should be noted that hysteresis effects [Möller et al., 2009) have been shown for repeated TMS pulses at the same stimulation site, potentially also affecting the proposed method by introducing an unheeded variance source. Here, a post-hoc split-half test did not identify significant differences for the localization across experimental time, supporting the validity of the realized randomization procedure. Future users of our localization method are advised to select coil positions/orientations in a quasi-random way and avoid stimulating multiple times successively in close proximity.

Here, we applied the proposed localization method in a domain that is characterized by a clear relationship between a single cortical site and the modulated behavioral effect. Due to its genericity, the method may be adapted to domains outside the motor cortex as long as i) a given, well circumscribed brain region is involved in a process that yields a measurable outcome variable and ii) the relationship between both can be modulated with TMS. Although we presented several monotonous and continuous functions to fit the dependent variable, the proposed method itself is not limited to these functional domains. Cognitive brain functions tend to feature a higher trial-by-trial variability and would thus require more stimulations than the 150 identified for the primary motor cortex. Here, the localization procedure was applied in healthy subjects. Functional localizations would in principle be possible in any brain region that produces a measurable outcome measure, thus excluding severely impaired regions for example after stroke. In addition, complex structure-function relationships for cognitive functions often rely on distributed networks of interacting regions and might involve a number of neighboring cortical patches instead of a single small region. Therefore, methodological extensions, such as multivariate regression approaches, are required to identify interdependent relationships between multiple neuronal populations.

Our approach was designed to increase the variance between electric field distributions via the use of random coil positions and orientations. This partially solves the problem of a missing criterion to define coil configurations a-priori (Weise et al., 2020), increases the potential information gain per pulse, and thus substantially reduces the required number of stimulations for a robust localization. Despite this efficacy, a further reduction of the number of stimulations might be feasible with optimization procedures, which identify optimal combinations of coil positions and orientations a priori. Allowing random coil positions and orientations instead of acquiring entire input-output curves for predefined coil positions/orientations establishes a highly efficient and robust experimental protocol.

In conclusion, we propose an efficient and easy to implement highresolution TMS localization method, applicable with standard TMS hardware. This framework enables the mapping of causal structurefunction relationships with a precision comparable to high-resolution neuroimaging techniques. Most importantly, the achieved mapping quality can be quantified, either online or post-experimentally. Our framework may be easily transferred to other functional domains with a single cortical representation.

\section{Declarations of Competing Interest}

The authors declare no conflicts of interest.

\section{Credit authorship contribution statement}

Ole Numssen: Conceptualization, Methodology, Software, Validation, Formal analysis, Investigation, Writing - original draft, Writing review \& editing, Visualization, Data curation. Anna-Leah Zier: Validation, Writing - original draft, Writing - review \& editing, Investigation, Software, Methodology. Axel Thielscher: Conceptualization, Methodology, Writing - review \& editing. Gesa Hartwigsen: Conceptualization,
Resources, Writing - review \& editing, Funding acquisition, Supervision. Thomas R. Knösche: Conceptualization, Writing - review \& editing, Funding acquisition, Supervision. Konstantin Weise: Conceptualization, Methodology, Software, Validation, Formal analysis, Investigation, Writing - original draft, Writing - review \& editing, Visualization, Data curation, Project administration.

\section{Acknowledgments}

This work was partially supported by the Deutsche Forschungsgemeinschaft (DFG) (HA 6314/3-1 and HA 6314/9-1 to GH, KN 588/101 to TRK and WE 59851/2 to KW); The Lundbeck Foundation (grants no. R244-2017-196 and R313-2019-622 to AT), the NVIDIA Corporation (donation of two Titan Xp graphics cards to GH and KW). GH is supported by the Max Planck Society.

\section{Data availability statement}

All electrical fields within the ROI and MEP data are made available at https://osf.io/jygu2/ .

\section{Supplementary materials}

Supplementary material associated with this article can be found, in the online version, at doi:10.1016/j.neuroimage.2021.118654.

\section{References}

Aberra, A.S., Wang, B., Grill, W.M., Peterchev, A.V., 2020. Simulation of transcranial magnetic stimulation in head model with morphologically-realistic cortical neurons. Brain Stimul. 13, 175-189. doi:10.1016/j.brs.2019.10.002.

Ahdab, R., Ayache, S.S., Farhat, W.H., Mylius, V., Schmidt, S., Brugières, P., et al., 2014. Reappraisal of the anatomical landmarks of motor and premotor cortical regions for image-guided brain navigation in TMS practice. Hum. Brain Mapp. 35, 2435-2447. doi:10.1002/hbm. 22339.

Ahdab, R., Ayache, S.S., Brugières, P., Farhat, W.H., Lefaucheur, J.-P., 2016. The hand motor hotspot is not always located in the hand knob: a neuronavigated transcranial magnetic stimulation study. Brain Topogr. 29, 590-597. doi:10.1007/s10548-016-0486-2.

Aonuma, S., Gomez-Tames, J., Laakso, I., Hirata, A., Takakura, T., Tamura, M., et al., 2018. A high-resolution computational localization method for transcranial magnetic stimulation mapping. Neuroimage 172, 85-93. doi:10.1016/j.neuroimage.2018.01.039.

Alavi, S.M.M., Goetz, S.M., Peterchev, A.V., 2019. Optimal estimation of neural recruitment curves using fisher information: application to transcranial magnetic stimulation. IEEE Trans. Neural Syst. Rehabil. Eng. 27, 1320-1330. doi:10.1109/tnsre.2019.2914475.

Bergmann, T.O., Lieb, A., Zrenner, C., Ziemann, U., 2019. Pulsed facilitation of corticospinal excitability by the sensorimotor $\mu$-alpha rhythm. J. Neurosci. 39, 10034 10043. doi:10.1523/jneurosci.1730-19.2019.

Bungert, A., Antunes, A., Espenhahn, S., Thielscher, A., 2017. Where does TMS stimulate the motor cortex? Combining electrophysiological measurements and realistic field estimates to reveal the affected cortex position. Cereb. Cortex 27, 5083-5094. doi:10.1093/cercor/bhw292.

Classen, J., Knorr, U., Werhahn, K.J., Schlaug, G., Kunesch, E., Cohen, L.G., et al., 1998. Multimodal output mapping of human central motor representation on different spatial scales. J. Physiol. 512 (Pt 1), 163-179. doi:10.1111/j.1469-7793.1998.163bf.x.

Dale, A.M., Fischl, B., Sereno, M.I., 1999. Cortical surface-based analysis. Neuroimage 9 , 179-194. doi:10.1006/nimg.1998.0395.

Diekhoff, S., Uludă̆, K., Sparing, R., Tittgemeyer, M., Cavuşoğlu, M., von Cramon, D.Y., et al., 2011. Functional localization in the human brain: gradient-Echo, Spin-Echo, and arterial spin-labeling fMRI compared with neuronavigated TMS. Hum. Brain Mapp. 32, 341-357. doi:10.1002/hbm.21024.

Dubbioso, R., Raffin, E., Karabanov, A., Thielscher, A., Siebner, H.R., 2017. Centresurround organization of fast sensorimotor integration in human motor hand area Neuroimage 158, 37-47. doi:10.1016/j.neuroimage.2017.06.063.

Dubbioso, R., Madsen, K.H., Thielscher, A., Siebner, H.R., 2021. The myelin content of the human precentral hand knob reflects interindividual differences in manual motor control at the physiological and behavioral level. J. Neurosci. 41 (14), 3163-3179. doi:10.1523/JNEUROSCI.0390-20.2021.

Dum, R., Strick, P., 2002. Motor areas in the frontal lobe of the primate. Physiol. Behav. 77, 677-682. doi:10.1016/s0031-9384(02)00929-0.

Fischl, B., Dale, A.M., Sereno, M.I., Tootell, R.B.H., Rosen, B.R., 1998. A coordinate system for the cortical surface. Neuroimage 7, S740. doi:10.1016/s1053-8119(18)31573-8.

Fox, P.T., Narayana, S., Tandon, N., Sandoval, H., Fox, S.P., Kochunov, P., et al., 2004 Column-based model of electric field excitation of cerebral cortex. Hum. Brain Mapp. 22, 1-14. doi:10.1002/hbm.20006.

Gaser C., Dahnke R., Kurth F., Luders E. A Computational Anatomy Toolbox for the Analysis of Structural MRI Data. NeuroImage n.d 2021. 
Geyer, S., Ledberg, A., Schleicher, A., Kinomura, S., Schormann, T., Bürgel, U., et al., 1996. Two different areas within the primary motor cortex of man. Nature 382, 805-807. doi:10.1038/382805a0

Gomez, L.J., Dannhauer, M., Peterchev, A.V., 2021. Fast computational optimization of TMS coil placement for individualized electric field targeting. Neuroimage 228, 117696. doi:10.1016/j.neuroimage.2020.117696.

Güllmar, D., Haueisen, J., Reichenbach, J.R., 2010. Influence of anisotropic electrical conductivity in white matter tissue on the EEG/MEG forward and inverse solution. A high-resolution whole head simulation study. Neuroimage 51, 145-163. doi:10.1016/j.neuroimage.2010.02.014.

Glasser, M.F., Coalson, T.S., Robinson, E.C., Hacker, C.D., Harwell, J., Yacoub, E., et al., 2016. A multi-modal parcellation of human cerebral cortex. Nature 536, 171. doi:10.1038/nature18933, -8

Goetz, S.M., Luber, B., Lisanby, S.H., Peterchev, A.V., 2014. A novel model incorporating two variability sources for describing motor evoked potentials. Brain Stimul. 7, 541552. doi:10.1016/j.brs.2014.03.002.

Goetz, S.M., Mahdi Alavi, S.M., Deng, Z.-.D., Peterchev, A.V, 2019. Statistical model of motor-evoked potentials. IEEE Trans. Neural Syst. Rehabil. Eng. 27, 1539-1545. doi:10.1109/tnsre.2019.2926543.

Goldsworthy, M.R., Hordacre, B., Ridding, M.C., 2016. Minimum number of trials required for within- and between-session reliability of TMS measures of corticospinal excitability. Neuroscience 320, 205-209. doi:10.1016/j.neuroscience.2016.02.012.

Huber, L., Finn, E.S., Handwerker, D.A., Bönstrup, M., Glen, D.R., Kashyap, S., et al., 2020. Sub-millimeter fMRI reveals multiple topographical digit representations that form action maps in human motor cortex. Neuroimage 208, 116463. doi:10.1016/j.neuroimage.2019.116463.

Kleim, J.A., Kleim, E.D., Cramer, S.C., 2007. Systematic assessment of training-induced changes in corticospinal output to hand using frameless stereotaxic transcranial magnetic stimulation. Nat. Protoc. 2, 1675-1684. doi:10.1038/nprot.2007.206.

Krieg, T.D., Salinas, F.S., Narayana, S., Fox, P.T., Mogul, D.J., 2013. PET-based confirmation of orientation sensitivity of TMS-induced cortical activation in humans. Brain Stimul. 6, 898-904. doi:10.1016/j.brs.2013.05.007.

Laakso, I., Murakami, T., Hirata, A., Ugawa, Y., 2018. Where and what TMS activates: experiments and modeling. Brain Stimul. 11, 166-174. doi:10.1016/j.brs.2017.09.011.

Martuzzi, R., van der Zwaag, W., Farthouat, J., Gruetter, R., Blanke, O., 2014. Human finger somatotopy in areas $3 \mathrm{~b}, 1$, and 2 : a 7T fMRI study using a natural stimulus. Hum. Brain Mapp. 35, 213-226. doi:10.1002/hbm.22172.

Mayka, M.A., Corcos, D.M., Leurgans, S.E., Vaillancourt, D.E., 2006. Threedimensional locations and boundaries of motor and premotor cortices as defined by functional brain imaging: a meta-analysis. Neuroimage 31, 1453-1474. doi:10.1016/j.neuroimage.2006.02.004.

Möller, C., Arai, N., Lücke, J., Ziemann, U., 2009. Hysteresis effects on the input-output curve of motor evoked potentials. Clin. Neurophysiol. 120 (5), 1003-1008.

Neggers, S.F.W., Langerak, T.R., Schutter, D.J.L.G., Mandl, R.C.W., Ramsey, N.F., Lemmens, P.J.J., et al., 2004. A stereotactic method for image-guided transcranial magnetic stimulation validated with fMRI and motor-evoked potentials. Neuroimage 21, 1805-1817. doi:10.1016/j.neuroimage.2003.12.006.

Ngomo, S., Leonard, G., Moffet, H., Mercier, C., 2012. Comparison of transcranial magnetic stimulation measures obtained at rest and under active conditions and their reliability. J. Neurosci. Methods 205, 65-71. doi:10.1016/j.jneumeth.2011.12.012.

Nielsen, J.F., 1996. Logarithmic distribution of amplitudes of compound muscle action potentials evoked by transcranial magnetic stimulation. J. Clin. Neurophysiol. 13, 423-434. doi:10.1097/00004691-199609000-00005.

Nielsen, J.D., Madsen, K.H., Puonti, O., Siebner, H.R., Bauer, C., Madsen, C.G., et al., 2018. Automatic skull segmentation from MR images for realistic volume conductor models of the head: assessment of the state-of-the-art. Neuroimage 174, 587-598. doi:10.1016/j.neuroimage.2018.03.001

Ninomiya, T., Inoue, K.-.I., Hoshi, E., Takada, M., 2019. Layer specificity of inputs from supplementary motor area and dorsal premotor cortex to primary motor cortex in macaque monkeys. Sci. Rep. 9. doi:10.1038/s41598-019-54220-z.

Opitz, A., Legon, W., Rowlands, A., Bickel, W.K., Paulus, W., Tyler, W.J., 2013. Physiological observations validate finite element models for estimating subject-specific electric field distributions induced by transcranial magnetic stimulation of the human motor cortex. Neuroimage 81, 253-264. doi:10.1016/j.neuroimage.2013.04.067.

Penny, W.D., Friston, K.J., Ashburner, J.T., Kiebel, S.J., Nichols, T.E., 2011. Statistical Parametric Mapping: The Analysis of Functional Brain Images. Elsevier.

Peterchev, A.V., Goetz, S.M., Westin, G.G., Luber, B., Lisanby, S.H., 2013. Pulse width dependence of motor threshold and input-output curve characterized with controllable pulse parameter transcranial magnetic stimulation. Clin. Neurophysiol. 124, 13641372. doi:10.1016/j.clinph.2013.01.011.
Raffin, E., Pellegrino, G., Di Lazzaro, V., Thielscher, A., Siebner, H.R., 2015. Bringing transcranial mapping into shape: sulcus-aligned mapping captures motor somatotopy in human primary motor hand area. Neuroimage 120, 164-175. doi:10.1016/j.neuroimage.2015.07.024.

Raffin, E., Siebner, H.R., 2019. Use-dependent plasticity in human primary motor hand area: synergistic interplay between training and immobilization. Cerebral. Cortex 29, 356-371. doi:10.1093/cercor/bhy226.

Rossi, S., Antal, A., Bestmann, S., Bikson, M., Brewer, C., Brockmöller, J., et al., 2021. Safety and recommendations for TMS use in healthy subjects and patient populations, with updates on training, ethical and regulatory issues: expert Guidelines. Clin. Neurophysiol. 132, 269-306. doi:10.1016/j.clinph.2020.10.003.

Rothwell, J.C., Hallett, M., Berardelli, A., Eisen, A., Rossini, P., Paulus, W., 1999. Magnetic stimulation: motor evoked potentials. The International Federation of Clinical Neurophysiology. Electroencephalogr. Clin. Neurophysiol. Suppl. 52, 97-103.

Saturnino, G.B., Madsen, K.H., Thielscher, A., 2019. Electric field simulations for transcranial brain stimulation using FEM: an efficient implementation and error analysis. J. Neural. Eng. 16, 066032. doi:10.1088/1741-2552/ab41ba.

Sarfeld, A.-.S., Diekhoff, S., Wang, L.E., Liuzzi, G., Uludağ, K., Eickhoff, S.B., et al., 2012. Convergence of human brain mapping tools: neuronavigated TMS parameters and fMRI activity in the hand motor area. Hum. Brain Mapp. 33, 1107-1123. doi:10.1002/hbm. 21272 .

Schaworonkow, N., Gordon, P.C., Belardinelli, P., Ziemann, U., Bergmann, T.O., Zrenner, C., 2018. $\mu$-Rhythm extracted with personalized eeg filters correlates with corticospinal excitability in real-time phase-triggered EEG-TMS. Front. Neurosci. 12. doi:10.3389/fnins.2018.00954.

Siebner, H.R., 2020. Does TMS of the precentral motor hand knob primarily stimulate the dorsal premotor cortex or the primary motor hand area? Brain Stimul. 13, 517-518. doi:10.1016/j.brs.2019.12.015.

Teitti, S., Määttä, S., Säisänen, L., Könönen, M., Vanninen, R., Hannula, H., et al., 2008. Non-primary motor areas in the human frontal lobe are connected directly to hand muscles. Neuroimage 40, 1243-1250. doi:10.1016/j.neuroimage.2007.12.065.

Tervo, A.E., Metsomaa, J., Nieminen, J.O., Sarvas, J., Ilmoniemi, R.J., 2020. Automated search of stimulation targets with closed-loop transcranial magnetic stimulation. Neuroimage 220, 117082. doi:10.1016/j.neuroimage.2020.117082.

Thielscher, A., Antunes, A., Saturnino, G.B., 2015. Field modeling for transcranial magnetic stimulation: a useful tool to understand the physiological effects of TMS? 2015 37th Annual International Conference of the IEEE Engineering in Medicine and Biology Society (EMBC) doi:10.1109/embc. 2015.7318340.

Triesch, J., Zrenner, C., Ziemann, U., 2015. Modeling TMS-induced I-waves in human motor cortex. Prog. Brain Res. 105-124. doi:10.1016/bs.pbr.2015.07.001.

Vaalto, S., Säisänen, L., Könönen, M., Julkunen, P., Hukkanen, T., Määttä, S., et al., 2011. Corticospinal output and cortical excitation-inhibition balance in distal hand muscle representations in nonprimary motor area. Hum. Brain Mapp. 32, 1692-1703. doi:10.1002/hbm.21137.

van de Ruit, M., Perenboom, M.J.L., Grey, M.J., 2015. TMS brain mapping in less than two minutes. Brain Stimul. 8, 231-239. doi:10.1016/j.brs.2014.10.020.

Veldema, J., Bösl, K., Nowak, D.A., 2017. Motor recovery of the affected hand in subacute stroke correlates with changes of contralesional cortical hand motor representation. Neural Plast. 2017, 6171903. doi:10.1155/2017/6171903.

Wagner, T., Gangitano, M., Romero, R., Théoret, H., Kobayashi, M., Anschel, D., et al., 2004. Intracranial measurement of current densities induced by transcranial magnetic stimulation in the human brain. Neurosci. Lett. 354, 91. doi:10.1016/s0304-3940(03)00861-9, -4.

Wassermann, E.M., Wang, B., Zeffiro, T.A., Sadato, N., Pascual-Leone, A., Toro, C., et al., 1996. Locating the motor cortex on the MRI with transcranial magnetic stimulation and PET. Neuroimage 3, 1-9. doi:10.1006/nimg.1996.0001.

Weise, K., Numssen, O., Thielscher, A., Hartwigsen, G., Knösche, T.R., 2020. A novel approach to localize cortical TMS effects. Neuroimage 209, 116486. doi:10.1016/j.neuroimage.2019.116486.

Wilson, S.A., Thickbroom, G.W., Mastaglia, F.L., 1993. Transcranial magnetic stimulation mapping of the motor cortex in normal subjects. J. Neurol. Sci. 118, 134-144. doi:10.1016/0022-510x(93)90102-5.

Yousry, T., 1997. Localization of the motor hand area to a knob on the precentral gyrus. A new landmark. Brain 120, 141-157. doi:10.1093/brain/120.1.141.

Zrenner, C., Desideri, D., Belardinelli, P., Ziemann, U., 2018. Real-time EEG-defined excitability states determine efficacy of TMS-induced plasticity in human motor cortex. Brain Stimul. 11, 374-389. doi:10.1016/j.brs.2017.11.016. 